УДК 616.895.8:159.972:616.89-008.42:57.018.634.725

Для цитирования: Сычугов Е.М., Касимова Л.Н., Зайцев О.С. Связь психопатологических особенностей у больных шизофренией с индивидуальным профилем функциональной асимметрии. Сибирский вестник психиатрии и наркологии. 2019; 1 (102): 28-36. https://doi.org/10.26617/1810-3111-2019-1(102)-28-36

\title{
Связь психопатологических особенностей у больных шизофренией с индивидуальным профилем функциональной асимметрии
}

\section{Сычугов Е.М., Касимова Л.Н., Зайцев О.С.}

Приволжский исследовательский медицинский университет Россия, 603005, Нижний Новгород, пл. Минина и Пожарского, дом 10/1

\section{PEЗЮME}

Статья посвящена поиску связи между функциональной асимметрией и психопатологическими особенностями при шизофрении. Имеющиеся данные касательно влияния индивидуального профиля функциональной асимметрии на клинические проявления шизофрении немногочисленны и носят зачастую противоречивый характер. Предполагается, что различные варианты функциональной асимметрии связаны с большей или меньшей частотой возникновения определённых психопатологических феноменов. Материалы: обследовано 46 пациентов с диагнозом шизофрении. Для каждого пациента был определён индивидуальный профиль функциональной асимметрии и проведена оценка особенностей клинической картины заболевания. Результаты: бредовые расстройства чаще отмечаются среди пациентов с правым и преимущественно правым профилем функциональной асимметрии. Галлюцинаторные и кататоно-гебефренные расстройства достоверно чаще встречаются в группе пациентов с симметричным и преимущественно левым профилем функциональной асимметрии. У пациентов с ведущим левым глазом чаще отмечаются полимодальность и комплексность галлюцинаций. Гипоманиакальные и маниакальные фазы достоверно реже возникают у пациентов с превосходством латерализации слуха над латерализацией руки. Выводы: обнаружена достоверная связь между психопатологическими особенностями у больных шизофренией и индивидуальным профилем функциональной асимметрии.

Ключевые слова: шизофрения, эндогенные психические расстройства, психопатология, функциональная асимметрия, церебральная латерализация.

\section{ВВЕДЕНИЕ}

Межполушарная асимметрия является объектом интереса со стороны неврологов со времён публикации фундаментальных работ Брока (1863), Дакса (1865) и Джексона (1876), которые продемонстрировали значимые различия между последствиями поражения того или иного полушария и соответствующими нарушениями речевых и ряда других когнитивных функций. В противоположность этому в психиатрии феномен латерализации церебральных функций до недавнего времени не вызывал особого интереса. Первые структурированные гипотезы относительно возможной взаимосвязи шизофрении с особенностями латерализации психических функций в головном мозге стали высказываться различными авторами с 1970-х годов $\mathrm{XX}$ века [1] и до настоящего времени продолжают подвергаться проверке с использованием всё возрастающего арсенала нейрофизиологических и нейробиологических методов исследования. Ряд авторитетных западных учёных считает обнаруживаемые при шизофрении отклонения от типичной мозговой латерализации ключом к пониманию этиопатогенетических основ данного заболевания [2, 3, 4, 5].
Среди теоретических концепций, рассматривающих шизофрению в качестве модели патологии мозговой латерализации и межполушарного взаимодействия, широкую известность получили работы T.J. Crow [6, 7]. Согласно выдвинутой им гипотезе, генетически обусловленная функциональная недостаточность правого полушария, лежащая в основе данного психического расстройства, приводит к компенсаторной гиперактивации левого полушария. Таким образом, эволюционно для этого не приспособленное левое полушарие головного мозга вынуждено взять на себя выполнение не только возросшего объёма задач, но и одновременное осуществление обработки информации двумя принципиально разными способами, что в итоге и приводит к специфической рассогласованности психической деятельности.

Однако современные исследования указывают на неполноценность подобного упрощённого подхода и предлагают фокусировать внимание на существенном ухудшении межполушарной координации (коммуникации) и общем ослаблении асимметрии функциональных связей у пациентов с шизофренией по сравнению со здоровыми испытуемыми $[8,9]$. 
Фенотипическим маркёром изменений мозговой латерализации психических функций выступает так называемый индивидуальный профиль функциональной асимметрии, определяемый как закономерное сочетание сенсорных, моторных и «психических» асимметрий, свойственных конкретному субъекту $[10,11]$.

Проблема зависимости между особенностями латерализации психических функций и конкретным клиническим типом шизофрении была рассмотрена в работе Nasrallah, McCalleyWhitters and Kuperman [12], которые утверждали, что только параноидная форма заболевания ассоциирована с нарушениями в мозговой латерализации и отсутствием доминанты по руке. Более поздние исследования подтвердили тезис, согласно которому среди параноидных пациентов амбидекстрия встречалась более часто [13]. Однако, согласно данным других авторов, наблюдаемая картина оказалась совершенно противоположнойю: по их результатам именно пациенты без параноидной симптоматики значительно чаще имели атипичную моторную латерализацию [14]. По сообщениям некоторых исследователей, отдельные симптомы заболевания также обнаружили определённую взаимосвязь с особенностями латерализации. В частности уменьшение латерализации по руке рассматривалось как предиктор таких достаточно специфичных симптомов шизофрении, как бред воздействия, трансляция мыслей на расстояние и симптом открытости $[15,16,17]$.

Вопрос зависимости психопатологической картины шизофрении от индивидуальных особенностей латерализации психических функций, фенотипически определяемых как сочетание моторных и сенсорных асимметрий, остаётся недостаточно изученным и требующим дальнейшей разработки.

\section{ЦЕЛЬ ИССЛЕДОВАНИЯ}

Целью исследования явилось выявление особенностей психопатологии шизофрении, феноменологии и синдромологии клинической картины заболевания в зависимости от индивидуального профиля функциональной асимметрии пациентов.

\section{МАТЕРИАЛЫ И МЕТОДЫ}

Для исследования была сформирована группа испытуемых, состоящая из 46 пациентов мужского пола в возрасте от 18 до 60 лет с диагнозом шизофрении, установленным согласно критериям МКБ-10, получавших психиатрическую помощь в стационарных и амбулаторных условиях на базе ГБУЗ НО ГКПБ № 1 г. Нижнего Новгорода.
Из исследования исключались пациенты с органическим поражением головного мозга, алкогольной и наркотической зависимостями, а также имевшие в анамнезе тяжёлые черепномозговые травмы либо установленные дефекты слуха и зрения.

Оценку индивидуального профиля функциональной асимметрии осуществляли посредством специально разработанного протокола, отражающего особенности моторной и сенсорной асимметрии. Протокол включал в себя самоопросник определения ведущей руки (Annet), «Hand Dominanz Test» (HDT) (оценивающий моторную асимметрию рук), моторные пробы А.Р. Лурия, тесты определения сенсорных асимметрий (проба Розенбаха, проба «Карта с дыркой» и др.), основанные на методических разработках Т.А. Доброхотовой, Н.Н. Брагиной, Е.Д. Хомской $[11,18]$. В анкету также были внесены результаты инструментального метода определения слухоречевой асимметрии - тест вербального дихотического прослушивания, предложенный Д. Кимурой [19] и адаптированный на русский язык Б.С. Котик [20]. В ходе данного теста испытуемому в наушники предъявлялось по 4 дихотические (различные на каждое ухо) пары односложных слов, после чего предлагалось назвать все услышанные им слова, затем процедура повторялась. Эксперимент был разделён на 2 серии по 9 предъявлений, между которыми наушники менялись местами во избежание аппаратных ошибок.

В процессе исследования определялись следующие показатели:

Коэффициент правой руки (КПР), оценивающий моторную асимметрию руки и рассчитываемый как:

$$
К П Р=(A+B) / 2 \text {, где }
$$

$\mathrm{A}$ - итоговый балл по тесту HDT, В - суммарный балл по опроснику Annet; абсолютные значения указанных величин были представлены в виде процентов.

Значения КПР> 20\% принимались как соответствующие правшеству, значения КПР $<-20 \%$ - левой асимметрии, значения $20 \geq К П Р \geq-20$ симметрии рук.

Коэффициент правого уха (КПУ), оценивающий степень асимметрии слуха в восприятии речи и рассчитываемый как:

$$
K \Pi У=(C-D) /(C+D) * 100, \text { где }
$$

$\mathrm{C}$ - количество слов, распознанных в дихотическом тесте правым ухом, D - количество слов, распознанных в дихотическом тесте левым ухом. 
Соотношение КПР/КПУ, позволяющее дифференцировать индивидуальный профиль функциональной асимметрии в соответствии с типологией, предложенной Т.И. Тетеркиной, и выносить суждение о наличии явления сенсомоторной диссоциации и инверсии [21].

Фенотипическая оценка индивидуального профиля асимметрии проводилась по четырём парным органам (рука, нога, ухо, глаз) посредством клинических проб, основанных на методических разработках отечественных авторов (А.Р. Лурия, Т.А. Доброхотовой, Н.Н. Брагиной, Е.Д. Хомской).
Преимущественное выполнение большинства проб по «правому» или по «левому» типу позволяло оценить показатель асимметрии каждого парного органа следующим образом: П (правый) - в случае преобладания «правых» проб, Л (левый) - в случае превалирования «левых» проб, А (без чёткой латерализации) в случае отсутствия «правой/левой» доминанты и невозможности однозначно оценить полученные показатели.

Суммарная оценка индивидуального профиля асимметрии, оцениваемого фенотипически, проводилась по пяти типам.

Т а бли ц а 1

Типы индивидуальных профилей функциональной асимметрии

\begin{tabular}{|c|c|c|c|c|c|}
\hline $\begin{array}{c}\text { Индивидуальный } \\
\text { профиль функцио- } \\
\text { нальной асимметрии }\end{array}$ & Правый профиль & $\begin{array}{c}\text { Преимуществен- } \\
\text { но правый про- } \\
\text { филь }\end{array}$ & $\begin{array}{c}\text { Симметричный } \\
\text { профиль }\end{array}$ & $\begin{array}{c}\text { Преимуществен- } \\
\text { но левый про- } \\
\text { филь }\end{array}$ & (ей профиль \\
\hline $\begin{array}{c}\text { Тип асимметрии } \\
\text { парных органов }\end{array}$ & (ПППП) & $(П П П *)$ & $(П \Pi * *)$ & $\left(\Pi^{* * *}\right)$ & $(* * * *)$ \\
\hline
\end{tabular}

П р и м е ч а н и е. П-правостороннее предпочтение, * - отсутствие правостороннего предпочтения (А, Л).

Оценка особенностей психопатологии осуществлялась клиническим методом посредством беседы с пациентом и клиникокатамнестическим методом, путём анализа медицинской документации и опроса медицинского персонала отделений.

Достоверность различий между группами устанавливалась по статистическим критериям в группах и хи-квадрат Пирсона для непараметрических данных.

\section{ОБСУЖДЕНИЕ РЕЗУЛЬТАТОВ}

В ходе суммарной фенотипической оценки, проводимой посредством клинических проб (не требующих особого инструментария и доступных для использования в практической работе любому клиницисту), профили функциональной асимметрии обследованных пациентов распределились следующим образом. $\mathrm{t}$ Стьюдента для сравнения средних значений

Правый тип профиля функциональной асимметрии наблюдался у 8 пациентов $(17,4 \%)$, преимущественно правый тип отмечался у 17 пациентов $(37, \%)$, симметричный - у 12 пациентов (26\%), преимущественно левый - у 9 пациентов $(19,6 \%)$. Левый тип среди обследуемых пациентов зафиксирован не был.

Обследованные пациенты с диагнозом шизофрении были подразделены на три клиникопсихопатологические группы, выделенные в зависимости от ведущего синдрома в клинической картине заболевания на всём его протяжении: 1) галлюцинаторный вариант параноидного синдрома, 2) бредовой вариант параноидного синдрома, 3) превалирование кататоногебефренных и полиморфных (лишённых синдромальной завершённости) расстройств. Проанализировав распространённость разных типов профилей функциональной асимметрии в каждой из групп, получены следующие данные. Т а б л и ц а 2

Распространённость типов профилей функциональной асимметрии в группах пациентов

\begin{tabular}{|l|c|c|c|c|c|}
\hline $\begin{array}{l}\text { Клинико-психопатологическая } \\
\text { группа }\end{array}$ & $\begin{array}{c}\text { Правый } \\
\text { профиль }\end{array}$ & $\begin{array}{c}\text { Преимуще- } \\
\text { ственно пра- } \\
\text { вый профиль }\end{array}$ & $\begin{array}{c}\text { Симметрич- } \\
\text { ный профиль }\end{array}$ & $\begin{array}{c}\text { Преимуще- } \\
\text { ственно левый } \\
\text { профиль }\end{array}$ & $\begin{array}{c}\text { Левый } \\
\text { профиль }\end{array}$ \\
\hline $\begin{array}{l}\text { Преимущественно галлюцина- } \\
\text { торные расстройства (n=16) }\end{array}$ & $1(6,25 \%)$ & $7(43,75 \%)$ & $3(18,75 \%)$ & $5(31,25 \%)$ & $0(0 \%)$ \\
\hline $\begin{array}{l}\text { Преимущественно бредовые } \\
\text { расстройства (n=17) }\end{array}$ & $6(35,30 \%)$ & $6(35,30 \%)$ & $5(29,40 \%)$ & $0(0 \%)$ & $0(0 \%)$ \\
\hline $\begin{array}{l}\text { Преимущественно кататоно- } \\
\text { гебефренические расстройства } \\
(\mathrm{n}=13)\end{array}$ & $1(7,69 \%)$ & $4(30,77 \%)$ & $4(30,77 \%)$ & $4(30,77 \%)$ & $0(0 \%)$ \\
\hline \multicolumn{1}{|c|}{ Итого } & $8(17,4 \%)$ & $17(37 \%)$ & $12(26 \%)$ & $9(19,6 \%)$ & $0(0 \%)$ \\
\hline
\end{tabular}


Как видно из представленных данных, в группе пациентов с превалированием в клинической картине заболевания бредовых расстройств доминировал правый и преимущественно правый тип профилей функциональной асимметрии. Напротив, преимущественно левый тип функциональной асимметрии в данной группе пациентов отсутствовал полностью. Как показано в целом, обнаруживается общая тенденция к росту доли симметричного и преимущественного левого профиля функциональной асимметрии в группах пациентов с превалированием галлюцинаторных и кататоногебефренных расстройств.

У 33 из 46 обследованных пациентов подтверждено наличие галлюцинаторных обманов восприятия в текущем психическом статусе или по данным катамнеза. 12 пациентов никогда галлюцинаторных обманов восприятия не испытывали. 1 пациент ввиду сомнительности и противоречивости предъявляемых в ходе исследования данных на предмет наличия или отсутствия галлюцинаторных обманов воспри- ятия в выборку по данному психопатологическому феномену включён не был. Следует отметить, что наличие или отсутствие галлюцинаций в клинической картине заболевания (равно как и остальных симптомов, рассматриваемых далее) не исключало возможности существования у того или иного пациента других психопатологических расстройств, оцениваемых в соответствующих рубриках. Анализ по каждому конкретному симптому в ходе исследования осуществлялся феноменологически с акцентом именно на качественные признаки расстройства, что позволило дифференцированно оценить зависимость отдельных психопатологических особенностей заболевания от индивидуального профиля функциональной асимметрии.

Согласно полученным в ходе исследования результатам, наличие «сенсомоторной диссоциации» обнаруживает достоверную связь с частотой возникновения галлюцинаторных обманов восприятия.

Т а б ли ц а 3

Связь между сенсомоторной диссоциацией и галлюцинациями

\begin{tabular}{|l|c|c|}
\hline \multicolumn{1}{|c|}{ Профиль функциональной асимметрии } & Без галлюцинаций & С наличием галлюцинаций \\
\hline Без сенсомоторной диссоциации & 8 & 5 \\
\hline С наличием сенсомоторной диссоциации & 4 & 28 \\
\hline
\end{tabular}

П р и м е ч а н и е. Критерий Хи-квадрат <0,001. Критерий Фишера р<0,05.

Связь между асимметрией по глазу и характером галлюцинаций

Т а б ли ц а 4

\begin{tabular}{|l|c|c|}
\hline \multicolumn{1}{|c|}{ Сенсорная асимметрия по глазу } & $\begin{array}{c}\text { Изолированный вербальный } \\
\text { псевдогаллюцино3 }\end{array}$ & $\begin{array}{c}\text { Полимодальность и/или галлюци- } \\
\text { нации других модальностей }\end{array}$ \\
\hline Правосторонняя & 13 & 11 \\
\hline Левосторонняя & 0 & 9 \\
\hline
\end{tabular}

П р и м е ч а н и е. Критерий Хи-квадрат 0,005. Критерий Фишера $<<0,05$.

Отдельного внимания заслуживает факт отсутствия предпочтения левого глаза среди пациентов, обнаруживавших изолированный от других обманов восприятия вербальный псевдогаллюциноз, что в сравнении с респондентами, чьи галлюцинаторные обманы восприятия касались других модальностей (зрительные, обонятельные, висцеральные) или характеризовались полимодальностью и комплексностью (до сценоподобного уровня), даёт статистически значимые различия. При предпочтении левого глаза отсутствовали наблюдения с изолированным (от других обманов восприятия) вербальным галлюцинозом, относительно часто встречались обманы восприятия других модальностей, а также полимодальные и комплексные галлюцинации. Психические автома- тизмы отмечались у 24 пациентов, отсутствие таковых имело место у 20. У 2 испытуемых имеющуюся симптоматику нельзя было достоверно классифицировать, вследствие чего они были исключены из данного феноменологического анализа. При сравнении индивидуальных профилей функциональной асимметрии было выявлено, что отсутствие в клинической картине психических автоматизмов статистически чаще наблюдается при предпочтении испытуемым левого глаза.

Наличие бредовых расстройств, регистрируемых в текущем психическом статусе на момент обследования или по данным катамнеза, было отмечено у 34 респондентов из 46. Наибольший интерес представляют результаты, согласно которым среди респондентов, не про- 
дуцировавших бредовых расстройств на протяжении заболевания, отсутствуют правши по руке. При попытке выявить зависимость типа бреда и его специфической фабулы от того или иного профиля функциональной асимметрии статистически достоверных различий получено не было, что может быть обусловлено недостаточным объёмом исследовательской выборки.

В представленных таблицах 5 и 6 проиллюстрированы полученные результаты.

Т а б ли ц а 5

\section{Связь между асимметрией по глазу и автоматизмами}

\begin{tabular}{|l|c|c|}
\hline \multicolumn{1}{|c|}{ Сенсорная асимметрия по глазу } & $\begin{array}{c}\text { Наличие } \\
\text { психических автоматизмов }\end{array}$ & $\begin{array}{c}\text { Отсутствие } \\
\text { психических автоматизмов }\end{array}$ \\
\hline Правосторонняя & 19 & 16 \\
\hline Левосторонняя & 1 & 8 \\
\hline
\end{tabular}

П р и м е ч а н и е. Критерий Хи-квадрат 0,021. Критерий Фишера р<0,05.

Связь между асимметрией по руке и бредовой симптоматикой

\begin{tabular}{|l|c|c|}
\hline \multicolumn{1}{|c|}{ Профиль моторной асимметрии руки } & $\begin{array}{c}\text { Наличие } \\
\text { бредовых расстройств }\end{array}$ & $\begin{array}{c}\text { Отсутствие } \\
\text { бредовых расстройств }\end{array}$ \\
\hline Правый профиль & 17 & 0 \\
\hline Левый и симметричный профиль & 17 & 12 \\
\hline
\end{tabular}

П р и м е ч а н и е. Критерий Хи-квадрат 0,003. Критерий Фишера р $<0,05$.

Отмечено, что в группе пациентов, у которых КПР менее латерализован, чем КПУ, достоверно реже отмечались маниакальные и гипоманиакальные фазы в текущем психическом статусе и по данным анамнеза. Какой-либо зависимости между проявлениями депрессивного синдрома и особенностями индивидуального профиля функциональной асимметрии в ходе исследования выявлено не было, что может быть обусловлено сложностью определения «чистых» аффективных фаз, на практике зачастую выступающих вторичными по отношению к другим психопатологическим расстройствам более высокого регистра.
Согласно данным литературы, у большинства психически здоровых испытуемых показатель КПУ (оценивающий степень латерализации слухоречевых функций) находится в интервале от -20 до 40. Именно эти значения принимались за условные границы нормы. Среди пациентов, испытывающих вербальный псевдогаллюциноз, показатель КПУ достоверно чаще превышал границы нормативного интервала, что в сравнении с группой пациентов, никогда подобных расстройств не переживавших, обнаружило статистически достоверные различия $(\mathrm{p}<0,05)$.

Связь между отношением КПР/КПУ и маниакальным аффектом

\begin{tabular}{|l|c|c|}
\hline \multicolumn{1}{|c|}{ Отношение КПР/КПУ } & $\begin{array}{c}\text { Наличие маниакальных и/или ги- } \\
\text { поманиакальных фаз в анамнезе }\end{array}$ & $\begin{array}{c}\text { Отсутствие маниакальных и/или } \\
\text { гипоманиакальных фаз в анамнезе }\end{array}$ \\
\hline $1 \leq$ КПР/КПУ $\leq 5$ & 10 & 13 \\
\hline $0<$ КПР/КПУ 1 & 2 & 15 \\
\hline
\end{tabular}

П р и м е ч а н и е. Критерий Хи-квадрат 0,031. Критерий Фишера р<0,05.

Т а б ли ц а 8

\section{Связь между значением КПУ и вербальным псевдогаллюцинозом}

\begin{tabular}{|l|c|c|}
\hline \multicolumn{1}{|c|}{ Показатель КПУ } & $\begin{array}{c}\text { Наличие вербального } \\
\text { псевдогаллюциноза }\end{array}$ & $\begin{array}{c}\text { Отсутствие вербального } \\
\text { псевдогаллюциноза }\end{array}$ \\
\hline Нормативные значения & 5 & 13 \\
\hline За границами нормативных значений & 17 & 11 \\
\hline
\end{tabular}

П р и м е ч а н и е. Критерий Хи-квадрат 0,030. Критерий Фишера р<0,05.

Выявленная взаимосвязь между бредовыми расстройствами и фенотипически определяемым профилем асимметрии согласуется с классической гипотезой T.J. Crow и работами современных авторов, согласно которым функциональная недостаточность правого полушария играет ключевую роль в механизмах бредообразования. По данным литературных публикаций, подобные наблюдения справедливы в отношении эндогенных психических заболеваний и органиче- 
ских поражений головного мозга. Рост числа правых и преимущественно правых профилей асимметрии среди пациентов с бредовыми расстройствами, вероятно, является фенотипическим отражением гиперактивации левого полушария головного мозга, возникающей компенсаторно в ответ на первичный функциональный дефицит правого. Но такую точку зрения разделяют не все авторы. В литературе приводятся данные, согласно которым пациенты с параноидной формой шизофрении в целом менее латерализованы по сравнению с контрольной группой. В попытке объяснить это противоречие имеет смысл говорить не о непосредственной связи клинической формы шизофрении с тем или иным типом асимметрии, а дифференцированно оценить зависимость бредовых расстройств от особенностей латерализации психических функций. В рамках данного подхода нами обнаружено, что бредовые расстройства в психопатологической картине заболевания, причем независимо от его клинической формы, отсутствуют в основном у пациентов с левосторонним или симметричным представительством моторной асимметрии руки. Статистически достоверной взаимосвязи между сенсорной асимметрией (зрение, слух) и бредовыми расстройствами не было выявлено в ходе исследования. Таким образом, увеличение удельной доли типичных правых профилей функциональной асимметрии, с особой значимостью правшества по руке, сопряжено с наличием бреда в психопатологической структуре заболевания, вне зависимости от его клинической формы.

Галлюцинаторные, кататоно-гебефренные и полиморфные, синдромально незавершённые расстройства, по данным исследования, достоверно чаще встречаются в группе пациентов с симметричным и преимущественно левым профилем функциональной асимметрии. У левшей, вероятно, существуют более сложные и нестабильные взаимоотношения между полушариями головного мозга и, как следствие, отмечается большее разнообразие психопатологических феноменов в случае возникновения шизофренического процесса. По нашим данным, галлюцинаторная симптоматика достоверно чаще наблюдается в случаях расхождения в предпочтениях моторных и сенсорных функций. Судя по всему, сенсомоторная диссоциация в индивидуальном профиле функциональной асимметрии также сопряжена с риском развития нарушений в межполушарном взаимодействии. Что же касается кататонической симптоматики, то увеличение её удельного веса в психопатологической структуре заболевания среди левшей и амбидекстров также может быть объяснено исходя из особенностей функциональной организации их головного мозга.

Давно установлен факт взаимосвязи кататонического синдрома в структуре шизофрении с перинатальными поражениями ЦНС, равно как и известных отклонений от типичной мозговой латерализации, достоверно регистрируемых уже на ранних этапах онтогенеза [22]. Отдельного внимания заслуживает роль левосторонней сенсорной асимметрии по глазу в отношении психопатологических феноменов. Псевдогаллюцинации у таких пациентов, помимо наиболее типичной для шизофрении слуховой модальности, отмечались в зрительной и тактильной сферах, часто формируя комплексный характер. Явления психических автоматизмов в данной группе обследованных, в свою очередь, встречались значительно реже, что заставляет думать о существовании своеобразных антагонистических отношений между этими феноменами. Однако патогенетическая основа подобных наблюдений остаётся неясной и ограничивается на данном этапе исследований лишь предположениями. В частности допустима гипотеза, согласно которой явления психических автоматизмов у пациентов с левосторонней функциональной асимметрией по зрению значительно чаще приобретают чувственную окрашенность, субъективно воспринимаясь и оцениваясь самим пациентом как псевдогаллюцинация той или иной модальности.

Анализ соотношения КПР и КПУ выявил взаимосвязь между наличием маниакальных и гипоманиакальных фаз в анамнезе и вариантом соотношений КПР/КПУ. В частности при превосходстве латерализованности слуха над латерализо-

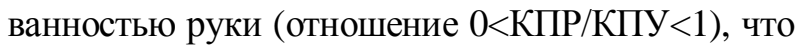
свойственно пациентам с дисфункцией правого полушария (по данным Т.И. Тетеркиной), достоверно реже отмечаются гипоманиакальные и маниакальные фазы в анамнезе.

Тем самым косвенно подтверждается ключевая роль изменения активности правого полушария головного мозга в генезе аффективных расстройств в рамках эндогенных психических заболеваний. Зарегистрированное увеличение показателей слухоречевой латерализации, в виде выхода показателей КПУ за границы принятых за «норму» значений, у пациентов с вербальным псевдогаллюцинозом соответствовало результатам предшествующих работ различных авторов [23]. 


\section{ЗАКЛЮЧЕНИЕ}

Бредовые расстройства чаще отмечаются среди пациентов с преимущественно правым и правым профилем функциональной асимметрии. Только у пациентов с левосторонним или симметричным типом моторной асимметрии по руке может отмечаться отсутствие бредовых расстройств в психопатологической картине шизофрении.

Галлюцинаторные и кататоно-гебефренные расстройства достоверно чаще встречаются в группе пациентов с симметричным и преимущественно левым профилем функциональной асимметрии. Кроме того, галлюцинаторные феномены достоверно чаще отмечаются при сенсомоторной диссоциации.

У пациентов с ведущим левым глазом часто отмечаются полимодальность и комплексность галлюцинаций и редко встречаются изолированный вербальный галлюциноз и психические автоматизмы.

Аффективные расстройства в виде гипоманиакальных и маниакальных фаз достоверно реже возникают у пациентов с превосходством латерализации слуха над латерализацией руки.

Превышение значения КПУ выше границ, принятых за норму, статистически чаще наблюдается среди пациентов, испытывающих вербальный псевдогаллюциноз.

\section{КОНФЛИКТ ИНТЕРЕСОВ}

Авторы заявляют об отсутствии возможных конфликтов интересов в связи с публикацией данной статьи.

\section{ИСТОЧНИК ФИНАНСИРОВАНИЯ}

Авторы заявляют об отсутствии финансирования при проведении исследования.

\section{СООТВЕТСТВИЕ ПРИНЦИПАМ ЭТИКИ}

Исследование проведено с соблюдением норм современной биомедицинской этики и этических стандартов, разработанных в соответствии с Хельсинской декларации ВМА (Протокол заседания этического комитета Приволжского исследовательского медицинского университета № 15 от 21.11.2016).

\section{ЛИТЕРАТУРА}

1. Flor-Henry P. Schizophrenic-like reactions and affective psychoses associated with temporal lobe epilepsy: etiological factors. Am J Psychiatry. 1969 Sep; 126 (3): 400-4. doi: 10.1176/ajp.126.3.400

2. Loberg E.M., Hugdahl K., Green M.F. Hemispheric asymmetry in schizophrenia: a "dual deficits" model. Biol Psychiatry. 1999; 45 (1):76-81.

3. Mitchell R.L., Crow T.J. Right hemisphere language functions and schizophrenia: the forgotten hemisphere? Brain. 2005. 128: 963-78. doi: 10.1093/brain/awh466
4. Paul L.K., Brown W.S., Adolphs R., Tyszka J.M., Richards L.J., Mukherjee P., Sherr E.H. Agenesis of the corpus callosum: genetic, developmental and functional aspects of connectivity. Nat Rev Neurosci. 2007; 8 (4): 287-99. doi: 10.1038/nrn2107

5. Hugdahl K., Løberg E.M., Jørgensen H.A., Lundervold A., Lund A., Green M.F., Rund B. Left hemisphere lateralization of auditory hallucinations in schizophrenia: a dichotic listening study. Cogn Neuropsychiatry. 2008. 13 (2): 166-79. doi: 10.1080/13546800801906808.

6. Crow T.J., Ball J., Bloom S.R., Brown R., Bruton C.J., Colter N., Frith C.D., Johnstone E.C., Owens D.G., Roberts G.W. Schizophrenia as an anomaly of development of cerebral asymmetry: a postmortem study and a proposal concerning the genetic basis of the disease. Arch Gen Psychiatry. 1989; 46 (12): 1145-1150. http://discovery.ucl.ac.uk/id/eprint/1516651

7. Crow T.J. Constrains on concepts of pathogenesis: Language and the speciation process as the key to the etiology of schizophrenia. Arch. Gen. Psychiatry. 1995; 52: 1011-4.

8. Hoptman M.J., Zuo X.N., D'Angelo D., Mauro C.J., Butler P.D., Milham M.P., Javitt D.C. Decreased interhemispheric coordination in schizophrenia: a resting state fMRI study. Schizophr. Res. 2012; 141 (1): 1-7. doi: 10.1016/j.schres.2012.07.027

9. Ribolsi M., Daskalakis Z.J., Siracusano A., Koch G. Abnormal asymmetry of brain connectivity in schizophrenia. Front. Hum. Neurosci. 2014; 8: 1010. doi: 10.3389/fnhum.2014.01010

10. Александров С.Г. Функциональная асимметрия и межполушарные взаимодействия головного мозга : учебное пособие для студентов. Иркутск: ИГМУ, 2014: 62.

11. Доброхотова Т.А. Нейропсихиатрия. Издание второе, исправленное. М.: Издательство БИHOM, 2013: 304.

12. Nasrallah A.H., McCalley-Whitters M., Kuperman S. Neurological differences between paranoid and nonparanoid schizophrenia: Part I. sensory-motor lateralization. Journal of Clinical Psychiatry. 1982; 48, 305-306.

13. Gureje O. Sensorimotor laterality in schizophrenia: Which features transcend cultural influences? Acta Psychiatrica Scandinavica. 1988; 77: 188-193.

14. Manschreck T.C., Ames D. Neurologic features and psychopathology in schizophrenic disorders. Biological Psychiatry. 1984; 19: 703-719.

15. Taylor P.J., Dalton R., Fleminger J.J. Handedness and schizophrenic symptoms. British Journal of Medical Psychology. 1982; 55 (3): 287-291.

16. Dollfus S., Buijsrogge J.A., Benalli K., Delamillieure P., Brazo P. Sinistrality in subtypes of schizophrenia. European Psychiatry. 2002; 17: 272-277.

17. Verdoux H., Liraud F., Droulout T., Theillay G., Parrot M., Franck N. Is the intensity of Schneiderian symptoms related to handedness and speech disorder in subjects with psychosis? Schizophrenia 
Research. 2004; 67: 167-173. doi: 10.1016/j.schres.2003.08.002

18. Хомская Е.Д. Нейропсихология: Учебник для вузов. 4-е издание. СПб.: Питер, 2014: 496.

19. Kimura D. Cerebral dominance and the perception of verbal stimuli. Canadian Journal of Psychology. 1961; 15 (3): 166-171.

20. Котик Б.С. Межполушарные взаимодействия при осуществлении речи у билингвов. Bonpocbl психологии. 1983; 6: 114-120.

21. Объедков В.Г., Тетеркина Т.И. Исходы шизофрении в контексте индивидуального профиля функциональной асимметрии головного мозга. Психическое здоровье. 2014; 9: 48-55.
22. Hepper P.G., McCartney G.R., Shannon E.A. Lateralized behaviour in first trimester human foetuses. Neuropsychologia. 1998; 36 (6): 531-534.

23. Malaspina D., Bruder G., Furman V., Gorman J.M., Berman A., Van Heertum R. Schizophrenia subgroups differing in dichotic listening laterality also differ in neurometabolism and symptomatology. J Neuropsychiatry. 2000 Fall; 12 (4): 485-92. doi: 10.1176/jnp.12.4.485

Поступила в редакцию 9.10.2018 Утверждена к печати 18.02.2019

Сычугов Евгений Михайлович, врач-психиатр, ассистент кафедры психиатрии и медицинской психологии. Касимова Лала Наримановна, доктор медицинских наук, профессор, заведующая кафедрой психиатрии и медицинской психологии.

Зайцев Олег Семенович, доктор медицинских наук, доцент кафедры психиатрии и медицинской психологии.

Сычугов Евгений Михайлович, sychugovem@gmail.com

УДК 616.895.8:159.972:616.89-008.42:57.018.634.725

For citation: Sychugov E.M., Kasimova L.N., Zaitsev O.S. Association of psychopathological features in schizophrenic patients with individual profile of functional asymmetry. Siberian Herald of Psychiatry and Addiction Psychiatry. 2019; 1 (102): 28-36. https://doi.org/10.26617/1810-3111-2019-1(102)-28-36

\section{Association of psychopathological features in schizophrenic patients with individual profile of functional asymmetry Sychugov E.M., Kasimova L.N., Zaitsev O.S.}

Federal State Budgetary Educational Institution of Higher Education «Privolzhsky Research Medical University» of the Ministry of Health of the Russian Federation

Minin and Pozharsky Square 10/1, 603005, Nizhny Novgorod, Russian Federation

\section{ABSTRACT}

Article is dedicated to search of association between functional asymmetries and psychopathology in schizophrenia. The available data concerning influence of an individual profile of functional asymmetry on clinical features of schizophrenia are not numerous and have often contradictory character. It is supposed that various types of functional asymmetry are associated with the frequency of occurrence of certain psychopathological phenomena. Materials: 46 patients with the diagnosis schizophrenia are examined. For each patient the individual profile of functional asymmetry is formed and the assessment of features of the clinical picture of the disease is carried out. Results: Delusional disorders are more often noted among patients with mainly right and right profile of functional asymmetry. Hallucinatory and catatonic/hebephrenic disorders reliably more often are found in group of patients with symmetric and mainly left profile of functional asymmetry. The polymodality and complexity of hallucinations is more often noted in patients with the leading left eye. Hypomaniacal and maniacal phases arise authentically less often in patients with superiority of lateralization of hearing over hand lateralization. Conclusions: Reliable association between psychopathological features of schizophrenia and individual profile of functional asymmetry is found.

Keywords: schizophrenia, endogenous mental disorders, psychopathology, functional asymmetry, cerebral lateralization.

\section{REFERENCES}

1. Flor-Henry P. Schizophrenic-like reactions and affective psychoses associated with temporal lobe epilepsy: etiological factors. Am J Psychiatry. 1969 Sep; 126 (3): 400-4. doi: 10.1176/ajp.126.3.400

2. Loberg E.M., Hugdahl K., Green M.F. Hemispheric asymmetry in schizophrenia: a "dual deficits" model. Biol Psychiatry. 1999; 45 (1):76-81.
3. Mitchell R.L., Crow T.J. Right hemisphere language functions and schizophrenia: the forgotten hemisphere? Brain. 2005. 128: 963-78. doi: 10.1093/brain/awh466

4. Paul L.K., Brown W.S., Adolphs R., Tyszka J.M., Richards L.J., Mukherjee P., Sherr E.H. Agenesis of the corpus callosum: genetic, developmental and 
functional aspects of connectivity. Nat Rev Neurosci. 2007; 8 (4): 287-99. doi: 10.1038/nrn2107

5. Hugdahl K., Løberg E.M., Jørgensen H.A., Lundervold A., Lund A., Green M.F., Rund B. Left hemisphere lateralization of auditory hallucinations in schizophrenia: a dichotic listening study. Cogn Neuropsychiatry. 2008. 13 (2): 166-79. doi: 10.1080/13546800801906808.

6. Crow T.J., Ball J., Bloom S.R., Brown R., Bruton C.J., Colter N., Frith C.D., Johnstone E.C., Owens D.G., Roberts G.W. Schizophrenia as an anomaly of development of cerebral asymmetry: a postmortem study and a proposal concerning the genetic basis of the disease. Arch Gen Psychiatry. 1989; 46 (12): 1145-1150. http://discovery.ucl.ac.uk/id/eprint/1516651

7. Crow T.J. Constrains on concepts of pathogenesis: Language and the speciation process as the key to the etiology of schizophrenia. Arch. Gen. Psychiatry. 1995; 52: 1011-4.

8. Hoptman M.J., Zuo X.N., D'Angelo D., Mauro C.J., Butler P.D., Milham M.P., Javitt D.C. Decreased interhemispheric coordination in schizophrenia: a resting state fMRI study. Schizophr. Res. 2012; 141 (1): 1-7. doi: 10.1016/j.schres.2012.07.027

9. Ribolsi M., Daskalakis Z.J., Siracusano A., Koch G. Abnormal asymmetry of brain connectivity in schizophrenia. Front. Hum. Neurosci. 2014; 8: 1010. doi: 10.3389/fnhum.2014.01010

10. Aleksandrov S. G. Funkcional'naya asimmetriya i mezhpolusharnye vzaimodejstviya golovnogo mozga : uchebnoe posobie dlya studentov [Functional asymmetry and interhemispheric interactions of the brain: manual for students]. Irkutsk: Irkutsk state medical University, 2014: 62 (in Russian).

11. Dobrohotova T.A. Nejropsihiatriya. Izdanie 2-e, ispravlennoe [Neuropsychiatry. Second edition, revised]. - Moscow: Izdatel'stvo BINOM, 2013: 304 (in Russian).

12. Nasrallah A.H., McCalley-Whitters M., Kuperman S. Neurological differences between paranoid and nonparanoid schizophrenia: Part I. sensory-motor lateralization. Journal of Clinical Psychiatry. 1982; 48, 305-306.

13. Gureje O. Sensorimotor laterality in schizophrenia: Which features transcend cultural influences? Acta Psychiatrica Scandinavica. 1988; 77: 188-193.
14. Manschreck T.C., Ames D. Neurologic features and psychopathology in schizophrenic disorders. Biological Psychiatry. 1984; 19: 703-719.

15. Taylor P.J., Dalton R., Fleminger J.J. Handedness and schizophrenic symptoms. British Journal of Medical Psychology. 1982; 55 (3): 287-291.

16. Dollfus S., Buijsrogge J.A., Benalli K., Delamillieure P., Brazo P. Sinistrality in subtypes of schizophrenia. European Psychiatry. 2002; 17: 272-277.

17. Verdoux H., Liraud F., Droulout T., Theillay G., Parrot M., Franck N. Is the intensity of Schneiderian symptoms related to handedness and speech disorder in subjects with psychosis? Schizophrenia Research. 2004; 67: 167-173. doi: 10.1016/j.schres.2003.08.002

18. Homskaya E.D. Nejropsihologiya: Uchebnik dlya vuzov. 4-e izd [Neuropsychology: The textbook for higher education institutions. Fourth edition]. Saint Petersburg: Piter, 2014: 496 (in Russian).

19. Kimura D. Cerebral dominance and the perception of verbal stimuli. Canadian Journal of Psychology. 1961; 15 (3): 166-171.

20. Kotik B.S. Mezhpolusharnye vzaimodejstviya pri osushchestvlenii rechi u bilingvov [Interhemispheric interactions at implementation of speech in bilinguals]. Voprosy psihologii - Journal of Psychology Problems. 1983; 6: 114-120 (in Russian).

21. Ob'edkov V.G., Teterkina T.I. Iskhody shizofrenii v kontekste individual'nogo profilya funkcional'noj asimmetrii golovnogo mozga [Schizophrenia outcomes in the context of an individual profile of functional asymmetry of a brain]. Psihicheskoe zdorov'e - Mental Health. 2014; 9: 48-55 (in Russian).

22. Hepper P.G., McCartney G.R., Shannon E.A. Lateralized behaviour in first trimester human foetuses. Neuropsychologia. 1998; 36 (6): 531-534.

23. Malaspina D., Bruder G., Furman V., Gorman J.M., Berman A., Van Heertum R. Schizophrenia subgroups differing in dichotic listening laterality also differ in neurometabolism and symptomatology. $J$ Neuropsychiatry. 2000; Fall; 12 (4): 485-92. doi: 10.1176/jnp.12.4.485

Received October 9.2018 Accepted February 18.2019

Sychugov Evgeny M., psychiatrist, assistant of the Department of Psychiatry and Medical Psychology, Privolzhsky Research Medical University, Nizhny Novgorod, Russian Federation.

Kasimova Lala N., MD, Prof., Head of the Department of Psychiatry and Medical Psychology, Privolzhsky Research Medical University, Nizhny Novgorod, Russian Federation.

Zaitsev Oleg S., MD, Associate Professor of psychiatry and medical psychology, Privolzhsky Research Medical University, Nizhny Novgorod, Russian Federation.

Sychugov Evgeny M., sychugovem@gmail.com 